\title{
Síndrome de boca ardiente. Eficacia de la aplicación tópica de capsaicina. Estudio piloto
}

\author{
León Espinosa S.*, López Jornet P.**, Frutos Ros R.*
}

\section{RESUMEN}

El síndrome de boca ardiente es un cuadro clínico complejo en el que el paciente manifiesta una sensación de escozor o ardor intrabucal sin que aparezcan lesiones clínicas objetivables. Objetivo: Valorar la eficacia mediante la aplicación de un gel de capsaicina a una concentración de 0.025 mg., en pacientes con síndrome de boca ardiente. Pacientes y método: Se estudiaron a un grupo de 29 pacientes a los que se les realiza un protocolo de recogida de datos que incluye anamnesis, historia médica, exploración bucal y pruebas complementarias. A 15 de dichos pacientes se les prescribe un gel de capsaicina y se les realiza un seguimiento durante varias semanas. Resultados: La eficacia de la capsaicina es relativamente baja, solo un $13 \%$ de los pacientes refiere una mejoría importante (con una disminución de mas de 3 puntos en las escalas analógico-visuales) y un $6 \%$ mejoría parcial (disminución menor de 3 puntos) con este tratamiento. Discusión: La estomatodinia es un cuadro clínico multifactorial que precisa de una mayor investigación tanto de su etiología como de su manejo terapéutico. Es de suma importancia realizar un correcto diagnóstico y explicar este al paciente para poder mantener unas metas realistas sobre las posibilidades de éxito.

Palabras clave: Síndrome de boca ardiente, capsaicina tópica, tratamiento.

\begin{abstract}
Burning mouth syndrome is a complex clinical condition, patients show a sensation of irritation or intraoral heat without objetivable clinical injuries. Objective: to value the effectiveness of a capsaicin 0,025\% gel application in patients with burning mouth syndrome. Patients and methods: 29 patients with burning mouth syndrome was examined by means of anamnesis, medical history, oral explortation and complementary tests. 15 of these patients were administered a capsaicin gel and were examined for several weeks. Results: the effectiveness of capsaicin is relatively low, only $13 \%$ of patients refer an important improvement (with a reduction more tha 3 points in the visual alalogical scales) and 6\% partial improvement ( reduction under 3 points) with this treatment. Discussion: burning mouth syndrome is a multifactorial clinical problem that needs a carefuly of its etiology and therapeutic management. It is of extreme importance make a correct diagnosis and explain this condition to the patient to be able to maintain realistic objectives on the success possibilities.
\end{abstract}

Key words: Burning mouth syndrome, topic capsaicin , treatment.

Aceptado para publicación: febrero 2004.

* Licenciado en Odontología.

** Médico estomatólogo. Prof. Titular Medicina Bucal. Universidad de Murcia.

León Espinosa S., López Jornet P., Frutos Ros R. Síndrome de boca ardiente. Eficacia de la aplicación tópica de capsaicina. Estudio Piloto. Av. Odontoestomatol 2004; 20-6: 297-304. 


\section{INTRODUCCIÓN}

La estomatodinia o síndrome de boca ardiente es un cuadro clínico complejo en el que el paciente manifiesta una sensación de escozor o ardor intrabucal sin que aparezcan lesiones clínicas objetivables.

La prevalencia se estima entre 0,7 - 15\% de la población (1). Aparece con más frecuencia en mujeres en edades medias-avanzadas de la vida, siendo típico en mujeres postmenopausicas. La proporción entre hombres y mujeres varía de un 3:1 a 9:1 según los autores $(2,3)$.

El principal síntoma de esta enfermedad es la presencia de dolor urente, quemante que suele aparecer de forma espontánea, aunque muchas veces el paciente nos refiere la coincidencia con un tratamiento odontológico previo. La sintomatología suele ser continua, aunque hay momentos en que se agudiza, se agrava (4-10).

Las localizaciones más frecuentes del síndrome de boca ardiente son la lengua (sobre todo la punta y los bordes), los labios (especialmente el inferior), el paladar y las encías (11).

Además de la sensación de ardor pueden acompañar a esta patología otros síntomas como alteraciones de la salivación (xerostomia, sensación de saliva espesa o filamentosa) así como alteraciones en el gusto, sabor amargo o metálico o combinaciones de ambos, halitosis, disfagia, etc. (2, 3, 12-14).

El tratamiento del síndrome de boca ardiente ha sido insatisfactorio durante mucho tiempo. Los diferentes tratamientos propuestos y ensayados han tratado de solucionar los hipotéticos factores etiológicos o simplemente han intentado disminuir o cesar los síntomas (10).

El objetivo de este estudio es valorar la eficacia de la capsaicina al 0,025mgr. en forma tópica de gel, siendo este uno de los tratamientos propuestos en la actualidad para el síndrome de boca ardiente.

\section{PACIENTES Y MÉTODO}

\section{Selección}

Un total de 29 pacientes que referían sintomatología de boca ardiente de forma continuada fueron examinados en la clínica odontológica de la Universidad de Murcia desde Enero hasta Octubre de 2003. Todos los pacientes dieron su consentimiento informado. A dichos pacientes se les realizó un protocolo de recogida de datos, elaborado previamente, con la finalidad de facilitar el diagnóstico. Este protocolo contiene la fecha de ingreso de los pacientes y datos de filiación, otro apartado dedicado a la historia medica. Se evalúan también los hábitos así como la medicación actual a la que están sometidos.

A continuación se procedió a realizar un cuestionario al paciente sobre las características de su patología, se registran los síntomas referidos y a que se asocia o atribuye; el tiempo de evolución, las características del dolor. Se recoge el modo de inicio, la localización, etc. Mediante una escala analógico-visual registramos la intensidad del ardor pudiendo ser de leve a severa. Se anoto también la evolución de los síntomas a lo largo del tiempo con el fin de poder clasificar a los pacientes, según los criterios de Lamey (11) si las molestias empeoran a lo largo del día, si aparecen de noche y si mejoran o desaparecen al comer o beber.

A su vez en este apartado se evaluó si el paciente presentaba otros síntomas asociados como pueden ser trastornos en el gusto, sequedad oral, etc.

El siguiente paso en la recogida de datos consistió en la exploración bucal que incluye un examen de la mucosa oral prestando particular atención al color y lubricación de la mucosa, a la presencia o ausencia de eritema, fisuraciones, ulceraciones, candidiasis, etc. Se inspeccionó la situación periodontal así como la situación dental, la oclusión y la situación protésica.

Además se revisó si existía algún tipo de trauma en la mucosa si había signos de mordisqueamiento, cúspides afiladas, malposiciones dentarias y la presencia de tics (movimientos labiales, succión, deglución atípica, interposición lingual u otros).

Entre las pruebas complementarias se realizo sialometria mediante drenaje y pruebas de laboratorio en las que se miden los niveles de glucosa, creatinina, urea, ácido úrico, hierro, colesterol, triglicéridos, bilirrubina, vit. B12, ácido fólico, hematíes, hemoglobi- 
na, hematocrito, VCM, HCM, plaquetas, VSG, leucocitos, GOT, GPT, GGT, fosfatasa, amilasa, Proteína T y anticuerpos antinucleares para descartar posibles enfermedades sistémicas.

\section{Criterios de exclusión}

Quedaron excluidos de este estudio 14 sujetos que refiriendo sensación de ardor y a los que tras realizarles el protocolo de recogida de datos y la correspondiente exploración bucal se les explico el objetivo y duración del tratamiento y rehusaron participar en él.

\section{Criterios de inclusión}

Fueron incluidos en este estudio 15 pacientes a los que tras haberles realizado una completa exploración, presentaban sensación de ardor o escozor intrabucal sin que aparezcan lesiones clínicas objetivables. A dichos pacientes se les explicó el objetivo y duración del estudio dando estos su consentimiento para participar. Este estudio ha seguido los principios de la Declaración de Helsinki

El trabajo se inicia con la aplicación tópica de la capsaicina en 15 pacientes con síndrome de boca ardiente. La capsaicina es un anestésico tópico extraído de la pimienta, que parece reducir la acumulación de diferentes neurotransmisores (sustancia P) a nivel de los nervios periféricos (21-26). Su administración produce por un lado una liberación brusca, aguda y dolorosa de sustancia P; y por otro lado una profunda y permanente disminución de la respuesta al dolor como consecuencia de la depleción obtenida de la sustancia P. Esta sustancia paradójicamente presenta como efecto secundario el ardor local por lo que en ocasiones es rechazado por los pacientes.

Se prescribe un gel de capsaicina $\left(\mathrm{Capsidol}^{\circledR}\right)$ a una concentración de 0,025 mg, que habrán de aplicarse dos veces al día: por la mañana, cuando no presentan síntomas o estos son mínimos, y por la tarde, coincidiendo con el pico de máximo ardor. Se le dan indicaciones para que apliquen el gel sobre aquellas zonas donde se localiza la sensación urente. Se expli- ca a los pacientes el efecto secundario que puede presentar la capsaicina y se les recomienda el uso de enjuagues con leche fría en el caso de que no puedan tolerar el ardor.

La duración total del estudio es de tres semanas, evaluándose a los pacientes a la semana y a las 3 semanas Los pacientes son evaluados antes de ser sometidos a tratamiento, durante este y una vez finalizado el tratamiento. Durante todo el periodo del estudio no podían utilizar ningún otro tratamiento para esta patología.

\section{Evaluación pretratamiento}

Antes de iniciar el tratamiento se llevo cabo un control de la placa bacteriana, se dieron a los pacientes instrucciones de higiene, se pulieron cúspides y se sustituyeron las restauraciones defectuosas. Para medir la intensidad de los síntomas se utilizan escalas analógicas visuales (EVA) considerándose:

- Leve si la puntuación se encuentra entre 1 y 3 puntos - Moderada si la puntuación oscila entre 4 y 7 puntos

- Severa si la puntuación es de 8 ó 9

- Extrema si el valor en la escala corresponde al 10.

\section{Evaluación durante el tratamiento}

Los individuos que participaron en el estudio fueron explorados a los siete días de su primera visita y a las dos semanas después del primer control.

Para la evaluación de los pacientes se elaboro un cuadernillo de seguimiento que nos permite valorar el grado de cumplimiento del tratamiento y la eficacia de la capsaicina.

\section{Evaluación después del tratamiento}

La evaluación final se realizó transcurridas tres semanas desde el inicio del tratamiento. En ella se lleva a cabo una completa exploración bucal y se responden las cuestiones del cuadernillo de seguimiento para posteriormente analizar los resultados y poder valorar la eficacia de la capsaicina en el tratamiento del síndrome de boca ardiente 


\section{RESULTADOS}

Del grupo original de 29 pacientes fue reducido a 15 sujetos de estudio (13 mujeres y 3 varones), con edades comprendidas entre los 46 y 80 años, estando el $73,3 \%$ comprendido entre los 50 y los 70 años.

Las principales patologías presentadas por los pacientes así como la medicación a la que se encuentran sometidos se presentan en las tablas $1 \mathrm{y}$ 2. La localización de los síntomas se muestra en la tabla 3, siendo la lengua la localización mas frecuente en un $93 \%$ de los casos. Los datos recogidos mediante la exploración oral, sialometria, índices de higiene y placa e índice CPTIN se presentan en la tabla 4, en la que observamos como un $80 \%$ de los pacientes presentan hipo salivación y un $53 \%$ refieren alteraciones del gusto.

Los resultados obtenidos una vez medidas las escalas visuales analógicas son los siguientes:

- Ninguno de los pacientes refiere la intensidad de sus síntomas como leve.

- El 73\% de los pacientes (11) presenta intensidad modera

- Dos de los pacientes (13\%) refiere intensidad severa en el momento de la recogida de datos inicial.

- Otros dos pacientes (13\%) señalan una intensidad extrema.

Al evaluar la evolución y ritmo diario de los síntomas se obtuvieron los siguientes resultados:

- En el 67\% de los pacientes las molestias eran mínimas al levantarse e iban empeorando a lo largo del día.

- Sólo un $13 \%$ de los sujetos manifestó tener la sintomatología de forma continua sin variaciones en su intensidad a lo largo del día.

- Un $13 \%$ de estos pacientes refieren mejoría o desaparición de los síntomas con la ingesta de comida o bebida.

Del grupo original de 29 pacientes fue reducido a 15 sujetos de estudio (13 mujeres y 3 varones), con edades comprendidas entre los 46 y 80 años, estando el $73,3 \%$ comprendido entre los 50 y los 70 años.
De los 15 pacientes que iniciaron el tratamiento 8 de ellos (53\%) deciden abandonarlo al acudir a la primera revisión, una semana después de prescribirles el preparado de capsaicina tópica, por no poder tolerar el picor que el producto les produce.

A excepción de un paciente que aunque se aplicó el preparado durante las tres semanas indico no haberlo hecho de manera correcta, dos veces al día, el resto de pacientes $(40 \%)$ concluyó correctamente el tratamiento.

Los resultados obtenidos de los 7 pacientes que realizaron el tratamiento durante las tres semanas de forma correcta son los siguientes:

- 2 de los pacientes (13,3\%), ambos de sexo femenino, obtuvieron una mejoría total pasando de una puntuación de 6.5 a 5 (en la primera semana) y 0.5 (en la tercera) y de 6.5 puntos a 4.5 a la semana y 2.5 al cabo de tres semanas. Refiriendo ambas pacientes síntomas muy leves.

- Solo uno de los pacientes $(6,7 \%)$ refiere mejoría parcial pasando de una puntuación de 6 a 4 puntos en ambos controles llevados a cabo a la semana de inicio y fin de tratamiento.

- El resto, 4 pacientes (26,6\%), no obtuvieron ninguna mejoría, obteniéndose en la valoración de las escalas analógico-visuales la misma puntuación o incluso mayor que al inicio del tratamiento.

\section{DISCUSIÓN}

Las causas y los mecanismos que conducen a este síndrome son todavía desconocidos, se considera multifactorial y podemos agruparlas en tres grupos de origen local, sistémico y psicológico $(4,12,13$, $16,17)$. Entre las causas locales debemos considerar todos aquellos factores que actúan irritando la mucosa bucal $(16,17)$.

Entre los factores sistémicos que podemos considerar en el origen del síndrome de boca ardiente estarían los cambios hormonales de la menopausia y el embarazo, los déficits vitamínicos, de hierro, zinc y folatos que pueden dar lugar a anemias de tipo ferropénicas, macrocíticas o perniciosas, alteraciones 
gastrointestinales (gastritis atróficas, ulcus péptico, colitis ulcerosa,...), neuropatías , alteraciones metabólicas ( trastornos lipidicos, hiper e hipotiroidismo, diabetes mellitus, etc.), alteraciones producidas por fármacos como antihipertensivos, hiperglucemiantes, $\beta$-bloqueantes, antiinflamatorios, entre otros $(16,17)$.

Muchos de los casos de estomatodinia se asocian a factores psicológicos $(4,18,19,20)$. Entre estos factores se encuentra la presencia de cuadros depresivos o ansiosos, determinados acontecimientos vitales como la pérdida de un ser querido, una separación o problemas laborales, conflictos emocionales, problemas de autoestima, hipocondriosis o incluso cancerofobia. En determinados pacientes se han descrito también trastornos psiquiátricos graves como psicosis (18).

El diagnostico y tratamiento son controvertidos, en términos en que no hay ningún test o instrumento de certeza, solo la ausencia de lesiones orales objetivables, por lo que es un procedimiento de exclusión. El tratamiento ha sido insatisfactorio durante mucho tiempo. Los diferentes tratamientos propuestos y ensayados han tratado de solucionar los hipotéticos factores etiológicos o simplemente han intentado disminuir o cesar los síntomas $(10,27)$.

La aplicación de capsaicina tópica ha sido utilizada para el tratamiento del dolor neuropatico y dolor de distribución facial. Epstein y Marcoe (21) fueron los primeros en utilizar la capsaicina de forma tópica en el síndrome de boca ardiente, obteniendo buenos resultados con la aplicación de capsaicina en forma de gel en dos pacientes con este síndrome.

Los resultados de este estudio nos indican que la capsaicina tiene una eficacia relativamente baja $(13,3 \%)$. Esta baja efectividad es debida en gran parte a sus efectos secundarios, el ardor que sigue a la aplicación local del preparado. Además seria necesario hacer estudios sobre su eficacia en muestras de mayor tamaño, durante un periodo de estudio más prolongado, con otro tipo de presentación o con concentraciones diferentes.

A pesar de su baja eficacia podemos considerarla de elección como tratamiento inicial en aquellos pacientes que presenten síndrome de boca ardiente ya que su aplicación tópica evita las interacciones y complicaciones que el uso de otros tratamientos sistémicos

En la actualidad, se están empleando numerosos fármacos psicoactivos para el tratamiento de la estomatodinia: los antidepresivos tríciclicos como la amitriptilina (dosis de 10 a 150 mg por día) o nortriptilina; los ansiolíticos como el diazepam y otro derivados benzodiacepinicos que han demostrado ser eficaces en determinados pacientes como demuestran los estudios realizados por Gorsky (9).

Femiano y colaboradores han realizado también estudios sobre los efectos del ácido alfa-lipoico (ALA), un antioxidante eficaz en la polineuropatía diabética, en el tratamiento de los síntomas del síndrome de boca ardiente observándose cierta eficacia pero siendo aún necesarios más estudios sobre dicha sustancia $(28,29,30)$.

Sardella y colaboradores han ensayado también con buches de clorhidrato de benzidamina al $0^{\prime} 15 \%$, un antiinflamatorio y analgésico, no encontrándose una eficacia significativa; al igual que ocurre con los enjuagues con anestésicos locales que han demostrado no ser un tratamiento eficaz por la corta duración del efecto analgésico (31).

Los resultados más prometedores hasta el momento se han obtenido de los estudios de Grushka, con la utilización de un derivado benzodiacepinico de acción anticonvulsionante, el clonazepam, a dosis de 0,25-1 mg/ día, consiguiéndose la reducción de la sensación de ardor en gran número de pacientes (32).

Petruzzi et al (33) describen la aplicación sistémica de capsaicina oral en un grupo de 25 pacientes, encontrando efectividad terapéutica en un corto periodo de tiempo, pero ocasionando problemas gastrointestinales en la gran mayoría de sus pacientes

En conclusión, es necesario seguir investigando en la etiología y manejo terapéutico de estos sujetos para mejorar su bienestar y calidad de vida, ya que existe un elevado numero de estos que no se benefician de los actuales tratamientos. Es de suma importancia saber diagnosticar correctamente a los 


\begin{tabular}{|l|c|}
\hline \multicolumn{2}{|c|}{ TABLA 1.- ANTECEDENTES MÉDICOS } \\
ENCONTRADOS EN ESTE ESTUDIO \\
\hline PATOLOGÍA & $N^{\circ}$ DE CASOS \\
\hline Problemas endocrinológicos: & \\
Hipotiroidismo & 5 \\
Hipercolesterolemia & 2 \\
Problemas neurológicos: & 1 \\
$\quad$ Parkinson & 1 \\
Depresión & 2 \\
Ansiedad & 2 \\
Problemas gastrointestinales: & 1 \\
Colon irritable & 1 \\
Colitis ulcerosa & 1 \\
Ulcera gástrica & 1 \\
Dispepsia & \\
Problemas cardiorrespiratorios: & 1 \\
Asma & 2 \\
HTA & \\
Alergias: & 2 \\
Polen & 1 \\
AINES, Yodo, penicilina & 1 \\
Otros: & \\
Algias & \\
Cefaleas/mareos & \\
Osteoporosis & \\
Artrosis & \\
Hábitos: & \\
Fumadores & \\
Ex-fumadores & \\
\hline
\end{tabular}

pacientes que acuden a nuestra consulta, intentando conocer los factores que influyen sobre su patología para poder modificarlos y establecer un protocolo de tratamiento adaptado para controlar dichos factores.

\section{BIBLIOGRAFÍA}

1. Zakrzewska JM. The burning mouth síndrome remains an enigma. Pain 1995; 62: 253-7.

2. Eguia del Valle A, Aguirre Urízar JM, MartínezConde R, Echebarria Goikouria MA, Sagasta Pujana O. Síndrome de boca ardiente en el País Vasco: estudio preliminar de 30 casos. Med Oral 2003; 8: 84-90.
TABLA 2.- TRATAMIENTO MÉDICO ACTUAAL AL QUE ESTABAN SOMETIDOS LOS PACIENTES

\begin{tabular}{|l|c|}
\hline TRATAMIENTO MÉDICO & $N^{\circ}$ DE CASOS \\
\hline Tranquilizantes (BZDs) & $8(53 \%)$ \\
Antidepresivos & $2(13 \%)$ \\
Tirosina & 2 \\
Tratamiento hormonal & \\
$\quad$ sustitutivo & 1 \\
Antihipertensivos & 2 \\
Parkinson & 1 \\
Calcio & 1 \\
Tratamiento xerostomía & 2 \\
\hline
\end{tabular}

\begin{tabular}{|l|c|}
\hline \multicolumn{2}{|c|}{ TABLA 3.- LOCALIZACIÓN DE } \\
LOS SÍNTOMAS \\
\hline LOCALIZACIÓN & $N^{\circ}$ DE CASOS \\
\hline Lengua & $93 \%$ \\
Bordes & $78,5 \%$ \\
Punta & $57 \%$ \\
Dorso & $36 \%$ \\
Vientre & $36 \%$ \\
Labio & $53 \%$ \\
Superior & $40 \%$ \\
Inferior & $40 \%$ \\
Paladar & $33 \%$ \\
Mucosa yugal & $33 \%$ \\
Encía & $33 \%$ \\
Otras (nariz, garganta, etc.) & $20 \%$ \\
\hline
\end{tabular}

TABLA 4.- RESULTADOS OBTENIDOS DE LA EXPLORACIÓN Y EXÁMENES COMPLEMENTARIOS

\begin{tabular}{|l|c|}
\hline DATOS ORALES & $N^{\circ}$ DE CASOS \\
\hline Trastornos del gusto & $53 \%$ \\
Hiposalivación & $80 \%$ \\
Higiene deficiente/Gingivitis & $27 \%$ \\
Enfermedad periodontal & $7 \%$ \\
Portador Prot. removible & $13 \%$ \\
Malposiciones dentarias & $13 \%$ \\
Parafunción: & $47 \%$ \\
$\quad$ Hábito empuje lingual & $14 \%$ \\
Mordiscos & $33 \%$ \\
$\quad$ Lengua fisurada/dentada & $20 \%$ \\
Tics & $20 \%$ \\
\hline Línea alba & \\
\hline
\end{tabular}


3. Grushka M. Clinical features of burning mouth síndrome. Oral Surg Oral Med Oral Pathol 1987; 63: $30-6$.

4. Silvestre Donat FJ, Serrano Martínez C. El síndrome de boca ardiente: revisión de conceptos y puesta al día. Medicina Oral 1997; 2: 30-8.

5. Tarkkila L , Linna M, Tintinen A, Lindqvist C, Meurman JH. Oral symptoms at menopausethe role of hormona replacement therapy. Oral Surg Oral Med Oral Pathol Oral Radiol \& Endod 2001; 92 (3): 276-80.

6. Silvestre Donat FJ. El síndrome de boca ardiente en el paciente geriátrico. En: Bullón Fernández P, Velasco Ortega E, eds. Odontoestomatología geriátrica. Coordinación Editorial IMEC: 271-80

7. Eguia del Valle A, Aguirre Urízar JM, Etxeberria Goikouria MA, Martínez-Conde Llamosas R. Síndrome de la boca ardiente: Aspectos epidemiológicos, etiopatogénicos, clínicos y terapéuticos. Revista Vasca de Odontoestomatología 2000; 10 (3): 33-49.

8. Rodrigo Rodríguez MA, García Núñez JA. Síndrome de boca ardiente. Gaceta dental 2000; 104: 86-8.

9. Gorsky M, Silverman S, Chinn H. Clinical characteristics and management outcome in the burning mouth syndrome. An open study of 130 patients. Oral Surg Oral Med Oral Pathol 1991; 72: 192-5.

10. Bergdahl J, Anneroth G. Burning mouth syndrome: literature review and model for research and management. J Oral Pathol Med 1993; 22: 4338.

11. Lamey PJ, Lamb AB. Lip component of burning mouth syndrome. Oral Surg Oral Med Oral Pathol 1994; 78: 590-3.

12. Hernández Marcos G, Herrera San Millán ML. Síndrome de la boca ardiente. Revisión y actualización bibliográfica. Revista Europea de Odontoestomatología 1998; 4: 245-9.
13. Chimenos E, Miravé M, Yagüe E. Síndrome de boca ardiente. Revisión, caso clínico y protocolo diagnostico. Archivos de odontoestomatología 1997; 13 (10): 637-42.

14. Caballero Herrera R. Estomatodinia: boca ardiente o quemante. Av Odontoestomatol 1999; 15: 35-40.

15. Lamb AB, Lamey PJ, REeve PE. Burning mouth syndrome: Psychological aspects. $\mathrm{Br}$ Dent $\mathrm{J}$ 1998; 165: 256-60.

16. Epstein Jb, Marcoe JH. Topical application of capsaicin for treatment of oral neuropathic pain and trigeminal neuralgia. Oral Surg Oral Med Oral Pathol 1994; 77: 135-40.

17. Ngom PI, Dubray C, Woda A. A human oral capsaicin pain model to assess topical anestheticanalgesic drugs. Neuroscience Letters 2001; 316 (3): 149-52.

18. Zhang WY, Wan Po A. The effectiveness of topically applied capsaicin. A meta-analysis. Eur J Clin Pharmacol 1994; 46: 517-22.

19. Volmink J, Lancaster T, Gray S, Silacy C. Treatments for postherpetic neuralgia: a Systematic review of randomized controlled trials. Family practice 1996; 13: 84-91.

20. Barclay L, Vega C. Capsaicin safe, effective for intractable pruritis ani. Gut 2003; 52: 1323-6.

21. Peñarrocha M, Martínez JM, Sanchis JM, eds. Anestesia local en odontología. Valencia. Ed. Promolibro; 1999 p. 58-9.

22. Bergdahl M, Bergdahl J. Burning mouth síndrome: prevalence and associated factors. J Oral Pathol Med 1999; 28: 350-4.

23. Maresky LS, Van der Bijl P, Gird I. Burning mouth síndrome. Evaluation of multiple variables among 85 patients. Oral Surg Oral Med Oral Pathol 1993; 75: 303-7.

24. Velasco Ortega E, Valencia Alejandre S, Blanco 
Carrión A, Velasco Ponferrada C. El síndrome de ardor bucal en el anciano. La identificación de los trastornos psíquicos en su etiopatogenia. Revista Española de Geriatría y Gerontología 1998; 91: 9119-24.

25. Paterson AJ, Lamb AB, Clifford TJ, Lamey PJ. Burning mouth syndrome: the relationship between the HAD scale and parafunctional habits. J Oral Pathol Med 1995; 24: 289-92.

26. Somacarrera Pérez ML, Pinos Sánchez H, Hernández G, Lucas-Tomas M. Síndrome de boca ardiente. Aspectos clínicos y perfil psicológico asociado. Archivos de Odontoestomatología 1998; 5: 299-306.

27. Scala A, Checchi L, Montevecchi M, Marini I, Giamberardino MA. Update on burning mouth syndrome: Overview and patient management. Crit Rev Oral Biol Med 2003; 14 (4): 275-91.

28. Femiano F, Gombos F, Scully C. Burning mouth síndrome(BMS): controlled open trial of the effcicy of alpha -lipoic acid (thioctid acid) on symptomatology. Oral diseases 2000; 6; 274-7.

29. Femiano F, Scully C, Gombos F. Idiopathic dysgeusia; an open trial of alpha lipoic acid (ALA) therapy. Int J Oral Maxillofac Surg 2002; 31: 625-8.

30. Femiano F, Scully C. Síndrome de boca ardiente. Estudio de la psicoterapia, medicación con ácido alfa-lipoico y combinación de terapias. Med Oral 2004; 9: 8-13.

31. Sardella A, Uglietti D, Demarosi F. Enjuagues orales con hidrocloruro de benzidamina en el manejo del síndrome de ardor bucal. Oral Surg Oral Med Oral Pathol Oral Radiol Endod 1999; 88: 683-6.

32. Grushka M, Epstein J, Mott A. An open-label dose escalation pilot study of the effect of clonazepam in burning mouth syndrome. Oral Surg Oral Med Oral Pathol Oral Radiol Endod 1998; 86: 557-61.

33. Petruzzi M, Lauritano D, De Benedittis M, Baldoni M, Serpico R. Systemic capsaicin for burning mouth síndrome: short-term results of a pilot study. J Oral Pathol Med 2004; 33: 111-4.

\section{CORRESPONDENCIA}

P. López Jornet

Hospital Morales Meseguer

Clínica Odontológica Medicina Bucal

Adv. Marques de los Vélez s/n

Murcia 30008

e-mail:majornet@um.es

TEL .968 230061

Fax 968239565 